\title{
CircANKRDII Knockdown Protects HPMECs from Cigarette Smoke Extract-Induced Injury by Regulating miR-I45-5p/BRD4 Axis
}

This article was published in the following Dove Press journal: International Journal of Chronic Obstructive Pulmonary Disease

\author{
Zheng Wang' \\ Yuqiang Zuo ${ }^{2}$ \\ Zhihong $\mathrm{Gao}^{2}$ \\ 'Department of Respiratory Medicine, \\ The Second Hospital of Hebei Medical \\ University, Shijiazhuang, Hebei, 050000, \\ People's Republic of China; ${ }^{2}$ Department \\ of Physical Examination Center, The \\ Second Hospital of Hebei Medical \\ University, Shijiazhuang, Hebei, 050000, \\ People's Republic of China
}

Background: Chronic obstructive pulmonary disease (COPD) is a major cause of death because of its high incidence and mortality, which is chiefly resulted from cigarette smoke exposure. A large number of studies show that circular RNA (circRNA) participates in regulating COPD process. This study aims to reveal the role of circRNA ankyrin repeat domain 11 (circANKRD11) in cigarette smoke extract (CSE)-induced cell apoptosis, inflammation, and oxidative stress.

Methods: The expression of circANKRD11, microRNA-145-5p (miR-145-5p) and bromodomain-containing 4 (BRD4) mRNA was detected by quantitative real-time polymerase chain reaction. The expression of apoptosis-related proteins and BRD4 protein was determined by Western blot. Cell apoptosis was detected by flow cytometry and Western blot. Cell inflammation was demonstrated by determining the levels of interleukin-1 $\beta$ (IL-1 $\beta$ ), IL-6 and tumor necrosis factor- $\alpha(\mathrm{TNF}-\alpha)$ through enzyme-linked immunosorbent assay. Oxidative stress was investigated by the reactive oxygen species (ROS) and malondialdehyde (MDA) determination assays as well as superoxide dismutase (SOD) activity assay. The binding relationship between miR-145-5p and circANKRD11 or BRD4 was predicted by circinteractome or MicroT_CDS online database, and identified by dual-luciferase reporter, RNA immunoprecipitation or RNA pull-down assay.

Results: CircANKRD11 and BRD4 expression were increased, whereas miR-145-5p expression was decreased in the lung tissues of smokers with or without COPD and CSEinduced HPMECs compared with the lung tissues of non-smokers as well as untreated HPMECs, respectively. CircANKRD11 silencing ameliorated CSE-induced cell apoptosis, inflammation, and oxidative stress. CircANKRD11 acted as a sponge of miR-145-5p, and regulated CSE-induced cell injury via sponging miR-145-5p. Additionally, miR-145-5p mimics protected against CSE-induced cell injury through targeting BRD4.

Conclusion: CircANKRD11 absence protected HPMECs from CSE-induced injury by regulating BRD4 through associating with miR-145-5p, which demonstrated that circANKRD11 had the potential to act as a diagnosis biomarker for smoker-caused COPD. Keywords: COPD, circANKRD11, miR-145-5p, BRD4

\section{Introduction}

Chronic obstructive pulmonary disease (COPD) is accompanied by respiratory symptom and is a leading cause of fatality rate globally. ${ }^{1}$ Chronic bronchiolitis and emphysema are two main features of COPD and are commonly resulted from virus infection as well as toxic particles or gases. ${ }^{2}$ Among the environmental
Department of Physical Examination

Center, The Second Hospital of Hebei

Medical University

Tel +86-03II-8704690I

Email tjzx1234556@I26.com
International Journal of Chronic Obstructive Pulmonary Disease 2021:16 887-899

887

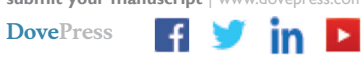

http://doi.org/10.2147/COPD.S3C 
factors, cigarette smoking (CS) is deemed to be the most vital one and CS-caused COPD patients account for more than $80 \%$ of all COPD suffers yearly. ${ }^{3}$ CS can indirectly cause endothelial cell injury, and then lead to the upregulation of cell apoptotic rate and oxidative stress. ${ }^{4,5}$ Nevertheless, the underlying mechanisms of CS-induced COPD have not been fully revealed.

Circular RNA is an evolutionarily conserved noncoding RNA and is found in various organisms, featured by high expression, stability and conservatism. ${ }^{6,7}$ Based on these characteristics, circRNA can be employed as a prognostic or diagnostic biomarker for many diseases. ${ }^{8}$ CircRNA is also involved in the incidence and development of pulmonary diseases. For instance, circRNA ankyrin repeat domain (ANKRD) 36 (circANKRD36) absence attenuated lipopolysaccharide (LPS)-triggered cell apoptosis and inflammation via hoisting microRNA-31-3p (miR-31-3p) in lung fibroblasts. ${ }^{9}$ Huang et al indicated that circ_0001953 and circ 0009024 were related to the severity of active tuberculosis. ${ }^{10}$ Zhou et al explained circ_0016070 contributed to cell growth, and thereby was correlated with pulmonary arterial hypertension by binding to miR-942. ${ }^{11}$ Additionally, circ_100395 $5^{12}$ and circ_0078767 ${ }^{13}$ were found to inhibit cell proliferative and invasive capacities in lung cancer. Nevertheless, there was little study on the mechanism of smoke-induced COPD mediated by circRNA. Our previous data showed circANKRD11 expression was increased in cigarette smoke extract (CSE)-treated pulmonary microvascular endothelial cells (HPMECs). Whether circANKRD11 regulated smoke-related COPD process was revealed in this study.

MiRNA is a small RNA and consists of about 20 nucleotides, regulating the expression of interest gene via binding to its non-coding region. ${ }^{14}$ A plenty of miRNAs are different in expression in the lung specimens between smokers with COPD and smokers without COPD. ${ }^{2,15}$ MiRNA also participates in regulating the pathogenesis of COPD by targeting mRNA. As reported by Diao et al miR-132 mimics upregulated the levels of interleukin-1 $\beta$ (IL-1 $\beta$ ) and tumor necrosis factor- $\alpha$ (TNF- $\alpha)$ through interacting with suppressor of cytokine signaling 5 (SOCS5) in human monocyte-like cells (THP1). ${ }^{16}$ MiR-543 was lowly expressed in the lung tissue samples of smokers with acute exacerbation COPD, and its overexpression could attenuate CSE-induced upregulation of interferon- $\gamma$ (INF- $\gamma$ ) and TNF- $\alpha$ via associating with fas apoptotic inhibitory molecule 2 (FAIM2). ${ }^{17}$ Bromodomain protein 4 (BRD4) belongs to Bromodomain and Extra-Terminal domain protein family and is an important regulator in gene transcription. ${ }^{18}$
Current researchers revealed that miR-145-5 $\mathrm{p}^{19}$ and BRD4 overexpression ${ }^{20}$ were involved in CSE-triggered cell apoptosis and inflammation in bronchial epithelial cells or HPMECs, suggesting miR-145-5p and BRD4 played vital parts in regulating CSE-induced COPD. Here we found that miR-145-5p possessed the complementary sequences of BRD4 and circANKRD11.

Thus, the impacts of circANKRD11 silencing on CSEinduced cell injury of HPMECs were revealed. The relationships among circANKRD11, miR-145-5p and BRD4 were disclosed. Furthermore, whether circANKRD11 modulated CSE-aroused HPMEC injury by miR-145-5p/ BRD4 pathway was unveiled.

\section{Materials and Methods Sample Collection and the Ethics Committee}

In accordance with the Declaration of Helsinki, the lung tissues from non-smokers ( $\mathrm{N}=10)$, smokers without COPD $(\mathrm{N}=17)$ and smokers with COPD $(\mathrm{N}=21)$ were collected from pneumonectomy suffers who underwent a surgery of solitary non-small cell lung cancer in the Second Hospital of Hebei Medical University. Obtained tissues were stored at $-80^{\circ} \mathrm{C}$ in a refrigerator. The written informed consent was signed by the subjects before operation. The Ethics Committee of the Second Hospital of Hebei Medical University approved this study. The clinical features of the subjects are listed in Table 1.

\section{CSE Preparation}

CSE was prepared based on the method shown by Richter and his colleagues. ${ }^{21}$ In brief, Liqun cigarettes (Tobacco Company; Xi'an, China) were combusted, and the smoke was bubbled through $25 \mathrm{~mL}$ Dulbecco's modified Eagle's medium (DMEM; Procell, Wuhan, China) with vacuum pumps (LeyNow, Dongguan, China). Then, the extract was filtered with $0.22 \mu \mathrm{m}$ filters (Millipore, Bradford, MA, USA). And the obtained solution was referred as $100 \%$ CSE and diluted to various concentrations $(0 \%, 0.5 \%, 1.0 \%, 2.5 \%$ and $5 \%$ ) with fetal bovine serum (FBS)-free DMEM (Procell).

\section{Cell Culture and CSE Treatment}

Human pulmonary microvascular endothelial cells (HPMECs) and human bronchial epithelioid cells were purchased from Otwo Biotech (Shenzhen, China), and cultured in DMEM (Procell) or Roswell Park Memorial Institute1640 (RPMI-1640; Procell) with 10\% FBS (Procell) and 
Table I Characteristics of Subjects in This Study

\begin{tabular}{|c|c|c|c|}
\hline & Non-Smokers & Smokers & Smokers with COPD \\
\hline Number & 10 & 17 & 21 \\
\hline Age (Years) & $61.3 \pm 3.1$ & $62.2 \pm 4.5$ & $64.5 \pm 5.8$ \\
\hline Male & $10(100 \%)$ & $17(100 \%)$ & $21(100 \%)$ \\
\hline Smoking history (pack-years) & 0 & $42.5 \pm 3.6 * *$ & $48.9 \pm 4.9 * * \#$ \\
\hline BMI $\left(\mathrm{kg} / \mathrm{m}^{2}\right)$ & $26.3 \pm 4.5$ & $28.9 \pm 4.2$ & $27.7 \pm 5.9$ \\
\hline $\mathrm{FEV}_{\text {, (L) }}$ & $3.5 \mathrm{I} \pm 0.11$ & $3.02 \pm 0.13$ & $2.08 \pm 0.12^{* \#}$ \\
\hline $\mathrm{FVC}(\mathrm{L})$ & $4.0 I \pm 0.33$ & $3.75 \pm 0.38$ & $3.66 \pm 0.15$ \\
\hline $\mathrm{FEV}_{\mathrm{l}} / \mathrm{FVC} \%$ & $81.02 \pm 1.2$ & $82.5 I \pm 1.5$ & $61.71 \pm 3.2 * * \#$ \\
\hline $\mathrm{FEV}_{\mathrm{I}}(\%$ predicted $)$ & $108.1 \pm 6.1$ & $96.7 \pm 3.8$ & $61.5 \pm 4.4 * * \#$ \\
\hline \multicolumn{4}{|l|}{ Occupational dust exposure, $\mathrm{n}$} \\
\hline Yes & I & 2 & 2 \\
\hline No & 9 & 15 & 19 \\
\hline \multicolumn{4}{|c|}{ Occupational hazard gas exposure, $\mathrm{n}$} \\
\hline Yes & 2 & I & I \\
\hline No & 8 & 16 & 20 \\
\hline
\end{tabular}

Notes: ${ }^{*} p<0.05$ and $*^{*} p<0.01$, different from non-smoker; $\# p<0.05$ and $\# \# p<0.01$, different from smokers.

Abbreviations: BMI, body mass index; FVC, forced vital capacity; $\mathrm{FEV}_{1}$, forced expiratory volume in one second.

$1 \%$ penicillin/streptomycin (Procell) at $37^{\circ} \mathrm{C}$ in an incubator with $5 \% \mathrm{CO}_{2}$. For CSE-related experiments, HPMECs were treated with CSE $(0 \%, 0.5 \%, 1.0 \%, 2.5 \%$ or $5 \%)$ and cultured for the defined time $(0,6,12,24$ or $36 \mathrm{~h})$.

\section{Cell Transfection}

The small interfering RNA against circANKRD11 (si-circANKRD11), miR-145-5p mimics (miR-145-5p), miR$145-5 \mathrm{p}$ inhibitors (in-miR-145-5p), and control groups (si-NC, miR-NC and in-miR-NC) were provided by GenePharma (Shanghai, China). The overexpression plasmid of BRD4 (BRD4), the wild-type (WT) vectors of circANKRD11 (circANKRD11 WT) and the 3'-untranslated region (3'UTR) of BRD4 (BRD4 3'UTR WT), the mutant (MUT) vectors of circANKRD11 (circANKRD11 MUT) and BRD4 3'UTR (BRD4 3'UTR MUT) and pcDNA $3.1(+)$ (pcDNA) were obtained from Geneseed Co., Ltd. (Guangzhou, China). Constructed plasmids or synthesized oligonucleotides mixed with TurboFect reagent (Thermo Fisher, Waltham, MA, USA) were incubated with wells according to the instruction of manufacture. The sequences of synthesized oligonucleotides were displayed as below. Si-circANKRD11 5'-GACACAG CAGACGGTTGATGA-3'; miR-145-5p 5'-GUCCAGUUU UCCCAGGAAUCCCU-3'; in-miR-145-5p 5'-AGGGAU UCCUGGGAAAACUGGAC-3'; si-NC 5'-CCTCTACCTGT CGCTGAGCTGTAAT-3'; miR-NC 5'-UUUGUACUAC ACAAAAGUACUG-3' and in-miR-NC 5'-CAGUACUU UUGUGUAGUACAAA-3'.

\section{Quantitative Real-Time Polymerase Chain Reaction (qRT-PCR)}

Collected lung tissues and HPMECs were split with TransZol (TransGen, Beijing, China), and RNA was isolated with an RNAsimple kit (Tiangen, Beijing, China). Then, cDNA was synthesized with a FastKing RT Kit (Tiangen) or MicroRNA Reverse Transcription Kit (Thermo Fisher). To quantity analysis of circANKRD11, miR-145-5p and BRD4, cDNA was mixed with primers and SuperReal PreMix Color (Tiangen), and reacted on a thermocycler (Thermo Fisher). The value of cycle threshold $(\mathrm{Ct})$ was assessed with the $2^{-\Delta \Delta \mathrm{Ct}}$ method with $\beta$-actin as a reference. The sequences of sense and antisense primers were circANKRD11 5'-GTGA GGGAGCGAGCCAGCAA-3' and 5'-ACCCTTGGGCA TCGTCCTG-3'; miR-145-5p 5'-ACACTCCAGCTGGG GTCCAGTTTTCCCAGGA-3' and 5'-TGGTGTCGTG GAGTCG-3'; BRD4 5'-CGTCTCCCTGACATTTCTTT-3' and 5'-GGTAATCCCTGGCAGTAGTT-3'; $\beta$-actin 5'-CAC CATTGGCAATGAGCGGTTC-3' and 5'-AGGTCTTTGC GGATGTCCACGT-3'.

\section{Annexin V-Fluorescein Isothiocyanate (Annexin V-FITC) and Propidium lodide (PI) Double Staining Assay}

To detect the apoptotic rate of HPMECs, Annexin V-FITC/ PI detection kit (Solarbio, Beijing, China) was employed. Briefly, HPMECs were collected after digestion with 
trypsin (Thermo Fisher). And cells were mixed with Binding Buffer (Solarbio) by vibrating. Then, Annexin V-FITC (Solarbio) and PI (Solarbio) were seriatim incubated with cells in dark. Samples were assessed with a flow cytometry (Thermo Fisher).

\section{Western Blot Analysis}

NP-40 buffer (Beyotime, Shanghai, China) was firstly employed to lyse cells, and lysates were mixed with loading buffer (Thermo Fisher). The mixture was then boiled in boiling water. After that, lysates were loaded by $15 \%$ bis-trisacrylamide gel (Thermo Fisher). The protein bands were electrotransferred onto nitrocellulose membranes (Membrane Solutions, Shanghai, China), which were then immersed in $8 \%$ defatted milk (Solarbio). Then, the membranes were incubated with primary antibodies against B-cell lymphoma-2 (Bcl-2) (1:1000; Abcam, Cambridge, UK), BCL2-associated x protein (Bax) (1:5000; Abcam), Cleaved caspase 3 (c-caspase 3; 1:5000; Abcam), pro-caspase 3 (p-caspase 3; 1:2000; Abcam), BRD4 (1:800; Abcam) and $\beta$-actin (1:1000; Abcam), respectively. Afterwards, secondary antibody against IgG H\&L (Abcam; 1:8000) was utilized to incubate the membranes. Finally, the protein bands were visualized by dropwise adding eyoECL Plus (Beyotime) onto the membranes. $\beta$-actin was employed as a control.

\section{Enzyme-Linked Immunosorbent Assay (ELISA)}

The levels of IL-1 $\beta$, IL- 6 and TNF- $\alpha$ were quantified by ELISA kits (Beyotime). In brief, cell supernatant was collected up by centrifuging at $300 \mathrm{~g}$ for $6 \mathrm{~min}$. Cell supernatant was diluted with dilution buffer (Beyotime) and added into reaction wells. Two hours later, reaction wells were washed, and incubated with biotinylated antibodies (Beyotime) for $1 \mathrm{~h}$. After that, streptavidin-marked horseradish peroxidase (Beyotime) and 3,3',5,5'Tetramethylbenzidine (TMB) were severally added into the wells and incubated in dark for $20 \mathrm{~min}$. The stop solution (Beyotime) was added, and samples were assessed using a microplate reader (Thermo Fisher) with the wavelength at $450 \mathrm{~nm}$.

\section{Reactive Oxygen Species (ROS) Detection Assay}

ROS level was detected by Cellular ROS Assay kit (Abcam). Briefly, HPMECs or CSE-treated HPMECs were cultured in 6-well plates and transfected with si-circANKRD11, si-NC,
in-miR-145-5p, in-miR-NC, miR-145-5p, miR-NC, BRD4 or pcDNA according the different purposes. Forty-eight hours later, cells were collected and seeded in clear bottom 96-well plates. After $24 \mathrm{~h}$, dichlorodihydrofluorescein diacetate (DCFH-DA) was utilized to stain cells in dark. The plates were measured by a fluorescence plate reader (Molecular Device, Sunnyvale, CA, USA) at excitation wavelength/emission wavelength $=485 \mathrm{~nm} / 535 \mathrm{~nm}$.

\section{Malondialdehyde (MDA) Determination}

\section{Assay}

MDA level was quantified by lipid peroxidation MDA assay kit (Abcam). In brief, cultured cells were harvested and washed using cold phosphate buffer solution (PBS; Solarbio). Then, cells were homogenized in Lysis Solution (Abcam), and insoluble material was removed by centrifuging. After that, thiobarbituric acid (TBA) was added into the wells and incubated at $95^{\circ} \mathrm{C}$ for $1 \mathrm{~h}$. Supernatant was assessed by measuring the wavelength at $532 \mathrm{~nm}$ using a microplate reader (Thermo Fisher).

\section{Superoxide Dismutase (SOD) Activity Detection}

SOD activity was detected by SOD activity assay kit (Abcam). In brief, grown HPMECs were collected and lysed with Tris/HCl (Millipore). Supernatant was harvested by centrifuging, and then placed on ice. Samples were mixed with Trifluoroacetic acid sodium salt (WST), Working Solution (Abcam) and Enzyme Working Solution (Abcam). SOD activity was determined by analyzing the output of wavelength at $450 \mathrm{~nm}$ with a microplate reader (Thermo Fisher).

\section{Dual-Luciferase Reporter Assay}

The binding sequence between miR-145-5p and circANKRD11 or BRD4 was severally predicted by circinteractome (https://circinteractome.nia.nih.gov/api/v2/ mirnasearch?circular rna query=hsa circ 0040929\&mirna_query $=\&$ submit $=$ miRNA + Target +Search) and MicroT_CDS online databases (http://diana. imis.athena-innovation.gr/DianaTools/index.php?r= microT_CDS/results\&keywords=hsa-miR-145-5p $\% 20 \%$ $20 \% 20$ E N G $00000141867 \&$ ge ne s $=$ ENSG00000141867\%20\&mirnas $=$ hsa-miR-145-5p\% $20 \&$ descr $=\&$ threshold $=0.1$ \&page $=1$ )..$\quad$ Built luciferase reporter plasmids were mixed with miR-145-5p or NC, and transfected into HPMECs with TurboFect reagent (Thermo Fisher). At the second day after transfection, 
cells were collected and firefly luciferase activity was quantified by Dual-Lucy Assay Kit (Solarbio) with Renilla luciferase activity as a control.

\section{RNA Immunoprecipitation (RIP) Assay}

RIP assay was conducted using a Magna RIP kit (Millipore). In brief, HPMECs were lysed with RIP buffer (Millipore) containing RNase inhibitor (Millipore). Then, lysates were incubated with antibody against argonaute2 (Anti-AGO2; 1:50; Abcam) and immunoglobulin G (AntiIgG; 1:100; Abcam), respectively. After $4 \mathrm{~h}$, magnetic protein $\mathrm{A} / \mathrm{G}$ beads (Millipore) were incubated with lysates for $24 \mathrm{~h}$. Then, proteinase $\mathrm{k}$ (Millipore) was added to digest proteins, and the contents of circANKRD11 and miR-145-5p were determined by qRT-PCR.

\section{RNA Pull-Down Assay}

HPMECs were seeded in 12-well plates and treated with biotinylated wild-type miR-145-5p (Bio-miR-145-5p WT; Sangon, Shanghai, China), biotinylated mutant miR-145-5p (Bio-miR-145-5p MUT; Sangon) and biotinylated NC (BioNC; Sangon), respectively. Cells were cultured for another 48 $\mathrm{h}$ and lysed with RIP buffer (Millipore). Then, streptavidincoupled beads (Invitrogen, Carlsbad, CA, USA) were incubated with the lysates. The magnetic beads were eluted, and the RNA complex pulled down was assessed by qRT-PCR.

\section{Statistical Analysis}

SPSS 21.0 software (IBM, Somers, NY, USA) was used to analyze the data derived from the three independent duplicate tests. Significant differences were compared with twotailed Student's $t$-tests between the two groups and with one-way analysis of variance (ANOVA) or Kruskal-Wallis test among three or more groups. The statistical significance was deemed when $P$ value $<0.05$.

\section{Results}

\section{CircANKRD I I Expression Was} Increased in the Tissues of COPD

\section{Patients and CSE-Induced HPMECs}

CircANKRD11 expression was firstly detected in the lung tissues of smokers without or with COPD. And qRT-PCR data showed circANKRD11 level was upregulated in smokers without COPD compared with non-smokers, and was higher in smokers with COPD than those without COPD (Figure 1A). This finding suggested that circANKRD11 expression might be correlated with the concentration of cigarette. To prove the guess, circANKRD11 expression was detected in CSE-induced HPMECs. Results presented that circANKRD11 expression was upregulated in a doseand time-dependent manner after treatment of CSE in HPMECs (Figure 1B and C). HPMECs were treated with $2.5 \% \mathrm{CSE}$ for $24 \mathrm{~h}$ in subsequent study. These results demonstrated that circANKRD11 might participate in regulating the process of smoke-caused COPD.

\section{CircANKRDI I Knockdown Attenuated CSE-Induced Cell Apoptosis, Inflammation, and Oxidative Stress in Pulmonary Endothelial Cells}

In order to reveal that whether circANKRD11 was enrolled in CSE-triggered lung injury, the effects between CSE and
A

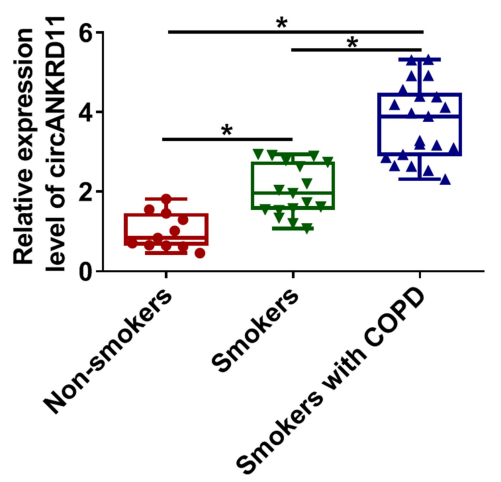

B

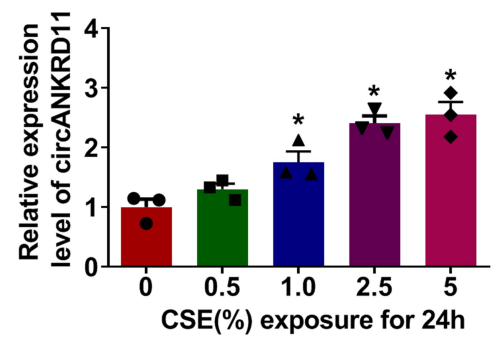

C

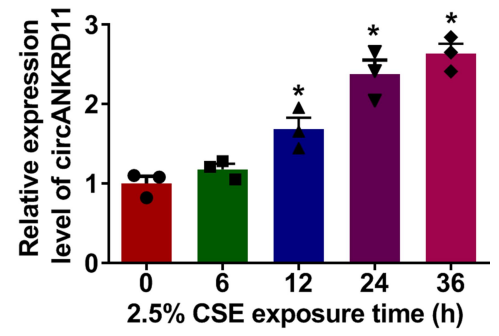

Figure I CircANKRDII was overexpressed in the tissues of COPD patients and CSE-induced HPMECs. (A) CircANKRDII expression was detected by qRT-PCR in the lung tissues of non-smokers $(N=10)$, smokers without COPD $(N=17)$ and smokers with COPD $(N=21)$. (B) CircANKRDII expression was determined by $q R T-P C R$ in HPMECs after treatment of CSE $(0 \%, 0.5 \%, 1.0 \%, 2.5 \%$ and $5 \%$ ) for $24 \mathrm{~h}$. (C) $2.5 \%$ CSE was exposed into HPMECs for $0,6,12,24$ and 36 h, respectively, and circANKRDII expression was determined by qRT-PCR. $* P<0.05$. 
circANKRD11 silencing on cell apoptosis, inflammation, and oxidative stress were firstly explored in HPMECs. Results initially presented that CSE treatment increased circANKRD11 expression, whereas this effect was reversed after circANKRD11 silencing (Figure 2A). Additionally, CSE exposure induced cell apoptosis, while circANKRD11 knockdown hindered this impact (Figure 2B). To further demonstrate the effects between CSE and circANKRD11 silencing on the apoptosis of HPMECs, the levels of apoptosis-related proteins (Bcl-2, Bax, c-caspase 3 and p-caspase 3) were detected. Western blot analysis exhibited that CSE treatment decreased Bcl-2 protein expression, and increased the protein level of Bax and the value of c-caspase 3/p-caspase 3; however, these effects were abolished by si-circANKRD11 (Figure 2C). Additionally, the production of IL-1 $\beta$, IL- 6 and TNF- $\alpha$ was promoted after treatment of CSE in HPMECs, but circANKRD11 knockdown impaired these effects (Figure 2D-F). Furthermore, CSE treatment upregulated the levels of ROS and MDA, and repressed SOD activity, which were reversed after circANKRD11 silencing in HPMECs (Figure 2G-I). Collectively, the above data demonstrated that circANKRD11 knockdown could ameliorate CSE-induced HPMEC injury.
Next, to determine whether the above observations could be repeatable in other human pulmonary endothelial cells, we studied the effects of circANKRD11 knockdown on inflammation and oxidative stress in CSE-treated HBE cells. Results initially presented the high efficiency of sicircANKRD11 in reducing circANKRD11 expression (Figure S1A). Additionally, results showed that the production of IL-1 $\beta$, IL- 6 , TNF- $\alpha$, ROS and MDA was promoted, and SOD activity was repressed after treatment of CSE in HBE cells, but circANKRD11 knockdown impaired these effects (Figure S1B-G). The above evidences demonstrated that circANKRD11 knockdown attenuated CSE-induced cell inflammation and oxidative stress in HBE cells. Based on the similar results in HPMECs and HBE cells, further experiments were performed in HPMECs.

\section{CircANKRDI I Acted as a Sponge of miR-I45-5p}

In order to reveal the mechanism of circANKRD11 in CSE-induced cell injury, the miRNA associated with circANKRD11 was continued to be explored. Circinteractome online database showed that miR-
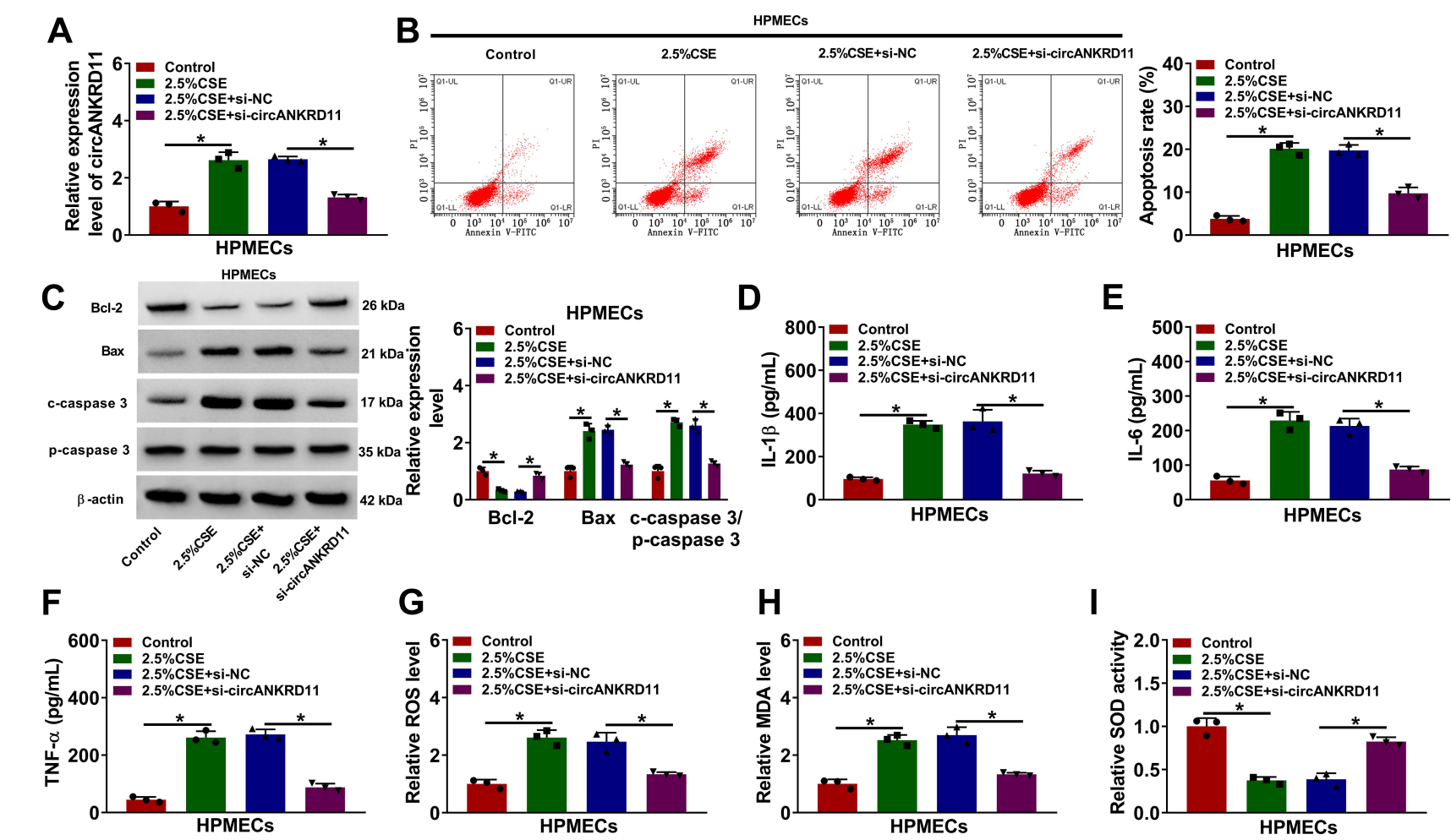

D
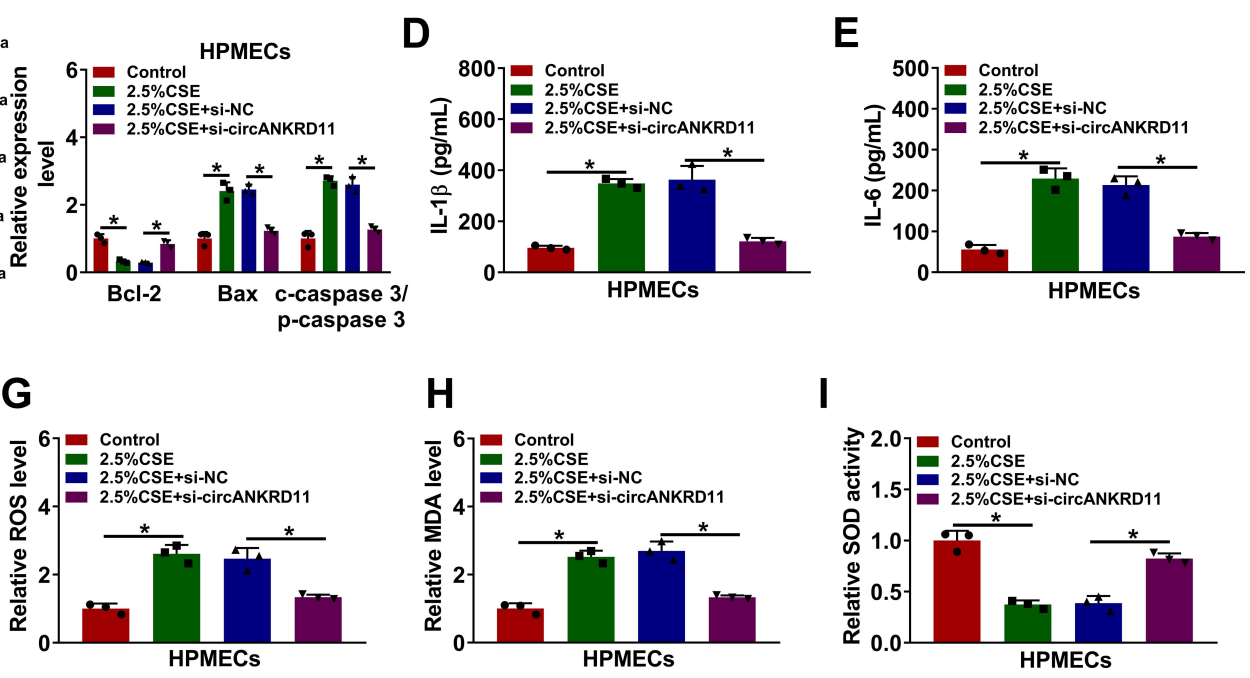

Figure 2 CircANKRDII absence protected HPMECs from CSE-induced injury. (A-I) HPMECs were treated with 0\% CSE (Control), $2.5 \%$ CSE, 2.5\% CSE+si-NC and 2.5\% CSE+si-circANKRDI I, respectively. (A) CircANKRDII expression was determined by qRT-PCR. (B) Cell apoptosis was detected by Annexin V-FITC and PI double staining assay. (C) The protein expression of Bcl-2, Bax, c-caspase 3 and p-caspase 3 was determined by Western blot analysis. (D-F) The levels of IL-I $\beta$, IL-6 and TNF- $\alpha$ were detected by ELISA. (G) The level of ROS was detected by ROS detection assay. (H) MDA level was detected by MDA determination assay. (I) SOD activity was detected by SOD activity detection assay. $* P<0.05$. 
$145-5 p$ possessed the binding sites of circANKRD11 (Figure 3A). To identify the binding relationship, the efficiency of synthesized miR-145-5p and in-miR-145$5 p$ in affecting miR-145-5p expression was firstly detected. The results from qRT-PCR presented that miR-145-5p expression was apparently upregulated by miR-145-5p mimics, and downregulated by miR-145$5 \mathrm{p}$ inhibitors (Figure 3B). Subsequently, dual-luciferase reporter assay showed the relative luciferase activity was dramatically repressed in the co-transfection group of circANKRD11 WT and miR-145-5p, but not in circANKRD11 MUT and miR-145-5p cotransfection group (Figure 3C). RIP assay displayed that circANKRD11 and miR-145-5p were significantly enriched in Anti-AGO2 group in comparison with Anti-IgG group in HPMECs (Figure 3D). Meanwhile,
RNA pull-down assay exhibited that circANKRD11 content was notably higher in Bio-miR-145-5p WT group than in Bio-miR-145-5p MUT group in HPMECs (Figure 3E). Additionally, qRT-PCR data presented that miR-145-5p expression was lower in the lung tissues of smokers without COPD than in nonsmokers, and was greatly lower in the lung tissues of smokers with COPD than in smokers without COPD (Figure 3F). MiR-145-5p expression was also decreased after treatment of CSE in HPMECs in comparison with untreated HPMECs (Figure 3G). Furthermore, miR-145-5p inhibitors also attenuated circANKRD11 silencing-mediated promotion impact on miR-145-5p expression in HPMECs (Figure 3H). Thus, the above data showed circANKRD11 was directly associated with miR-145-5p in HPMECs.
A

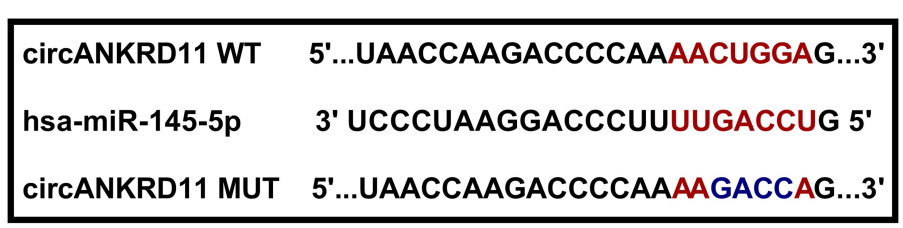

C

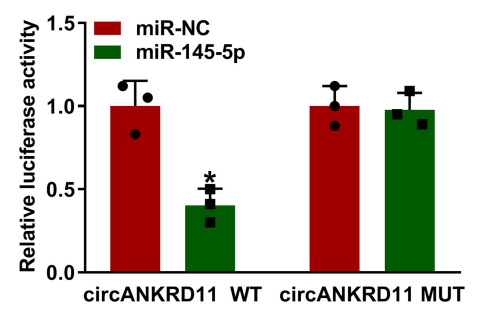

$\mathbf{F}$

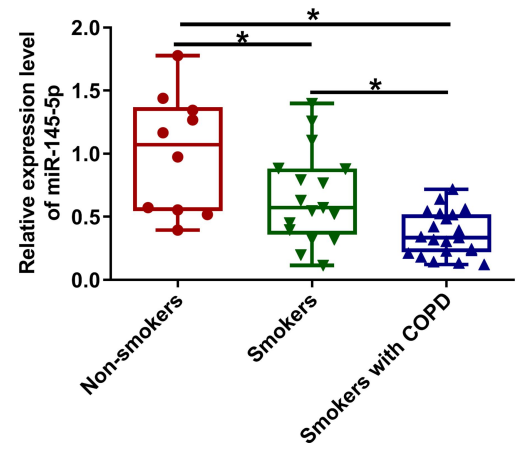

D

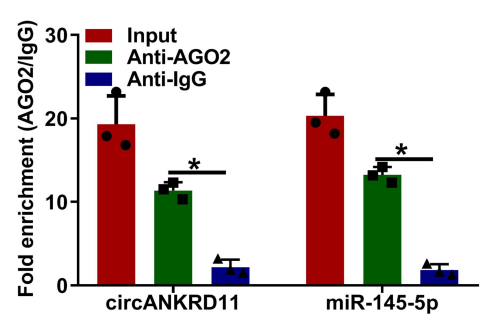

G

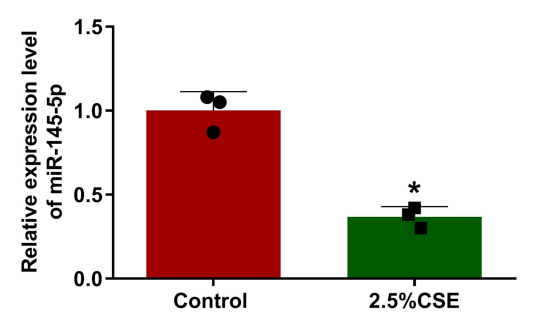

B

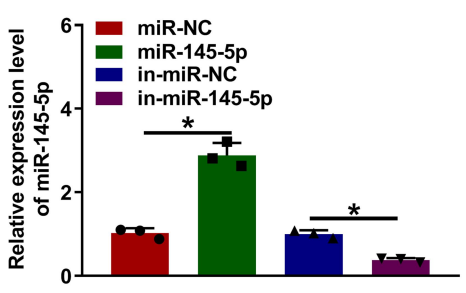

$\mathbf{E}$
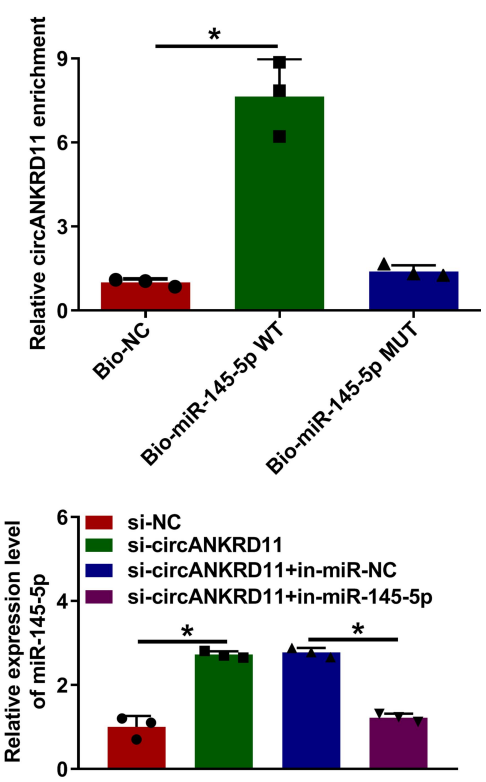

Figure 3 CircANKRDI I directly bound to miR-I45-5p in HPMECs. (A) Circinteractome online database was employed to predict the binding sites between circANKRDI I and miR-145-5p. (B) MiR-145-5p expression was detected by qRT-PCR in HPMECs transfected with miR-NC, miR-145-5p, in-miR-NC or in-miR-145-5p. (C-E) Dualluciferase reporter, RIP and RNA pull-down assays were employed to demonstrate the binding relationship between circANKRDII and miR-I45-5p in HPMECs. (F) MiRI45-5p expression was detected by qRT-PCR in the lung tissues of non-smokers ( $N=10)$, smokers without COPD ( $N=17)$ and smokers with COPD ( $N=21$ ). (G) MiR-I45-5P expression was determined by qRT-PCR in $2.5 \%$ CSE-treated HPMECs and untreated HPMECs. $(\mathbf{H})$ The impacts between circANKRDII silencing and miR-I45-5P inhibitors on miR-145-5p expression were determined by qRT-PCR in HPMECs. $* P<0.05$. 


\section{CircANKRD I I Regulated CSE-Induced} Cell Apoptosis, Inflammation, and Oxidative Stress by Sponging miR-145-5p Given the binding relationship between circANKRD11 and miR-145-5p, whether miR-145-5p participated in circANKRD11-mediated effects on CSE-caused cell injury was revealed in this part. Results showed miR-145-5p inhibitors reversed circANKRD11 silencing-mediated repressive impact on cell apoptosis in CSE-induced HPMECs (Figure 4A). Meanwhile, circANKRD11 knockdown upregulated $\mathrm{Bcl}-2$ protein expression, and downregulated Bax protein expression and the value of c-caspase 3/p-caspase 3 ; however, these impacts were impaired by miR-145-5p inhibitors (Figure 4B). The repressive impacts of circANKRD11 knockdown on the production of IL-1 $\beta$, IL- 6 and TNF- $\alpha$ were also attenuated after transfection of in-miR-145-5p (Figure 4C-E). Furthermore, circANKRD11 absence downregulated the levels of ROS and MDA, and promoted SOD activity, whereas these effects were abolished by miR-145-5p inhibitors (Figure
4F-H). Thus, these evidences demonstrated that circANKRD11 modulated CSE-induced HPMEC injury by binding to miR-149-5p.

\section{CircANKRDI I Regulated BRD4 Expression by Interacting with miR-145- $5 p$}

The target gene of miR-145-5p was continued to be sought. MicroT_CDS online database presented that BRD4 3'UTR contained the binding sites of miR-145$5 \mathrm{p}$ (Figure 5A). Subsequently, dual-luciferase reporter assay displayed that the relative luciferase activity was apparently inhibited in BRD4 3'UTR WT and miR-145$5 \mathrm{p}$ co-transfection group, but not in BRD4 3'UTR MUT and miR-145-5p co-transfection group (Figure 5B). The results from qRT-PCR displayed high mRNA and protein expression of BRD4 in the lung tissues of smokers with COPD compared with non-smokers, and higher mRNA and protein expression of BRD4 in the lung tissues of

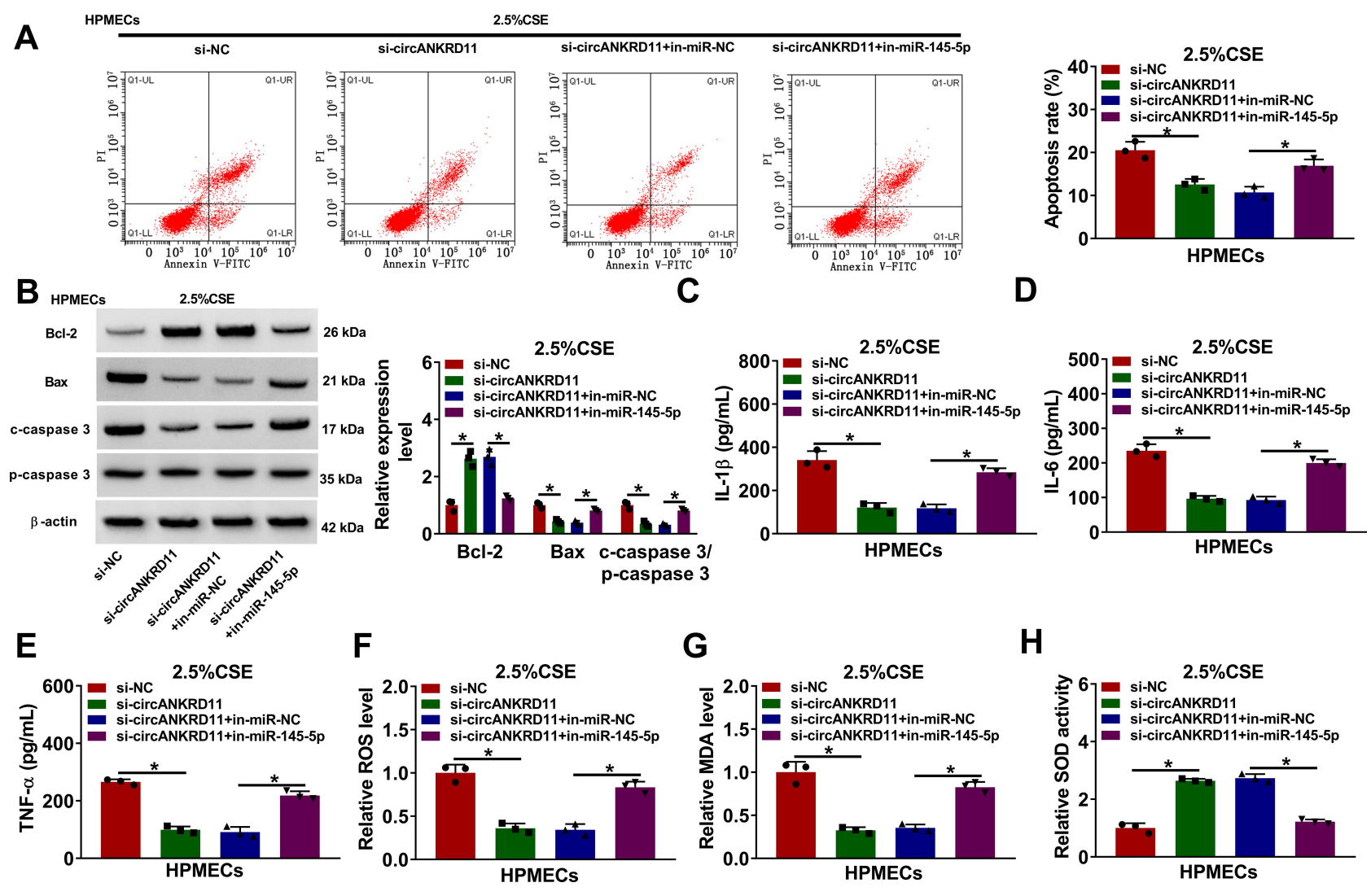

Figure 4 CircANKRDII knockdown repressed cell apoptosis, inflammation, and oxidative stress by interacting with miR-I45-5p in CSE-induced HPMECs. (A-H) $2.5 \%$ CSE-induced HPMECs were severally transfected with si-NC, si-circANKRDI I, si-circANKRDI I+in-miR-NC and si-circANKRDI I+in-miR-I45-5p. (A) Annexin V-FITC and PI double staining assay was employed to detect cell apoptosis. (B) The protein levels of Bcl-2, Bax, c-caspase 3 and p-caspase 3 were determined by Western blot analysis. (C-E) The levels of IL-I $\beta$, IL-6 and TNF- $\alpha$ were detected by ELISA. (F) The level of ROS was detected by ROS detection assay. (G) MDA level was detected by MDA determination assay. $(\mathbf{H})$ SOD activity was detected by SOD activity detection assay. $* P<0.05$. 
A

\begin{tabular}{lc|}
\hline BRD4 3'UTR WT & 5'..CCUGCCUUCUUCUGGAACUGGAA...3' \\
hsa-miR-145-5p & 3' UCCCUAAGGACCCUUUUGACCUG 5' \\
BRD4 3'UTR MUT & 5'...CCUGCCUUCUUCUGGAAGACGAA...3'
\end{tabular}

B

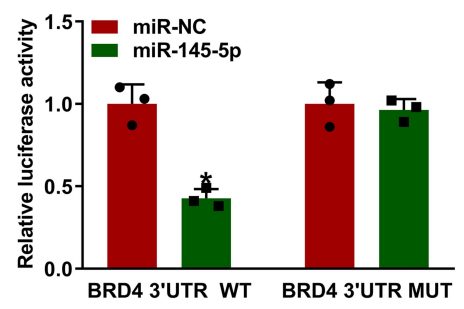

C

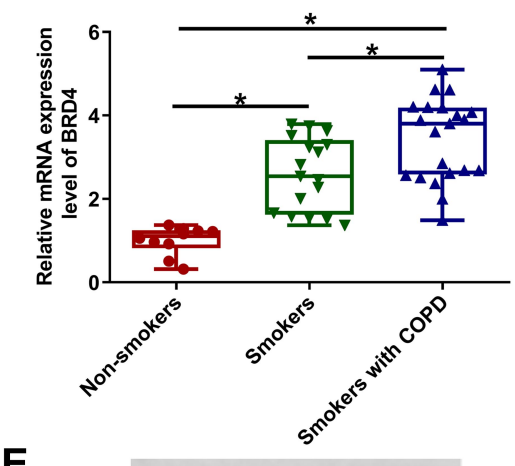

E

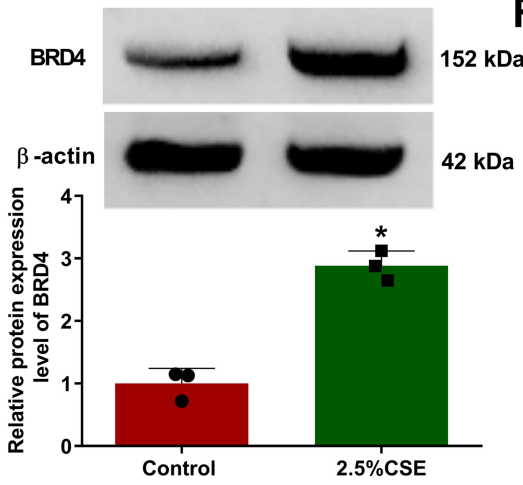

D
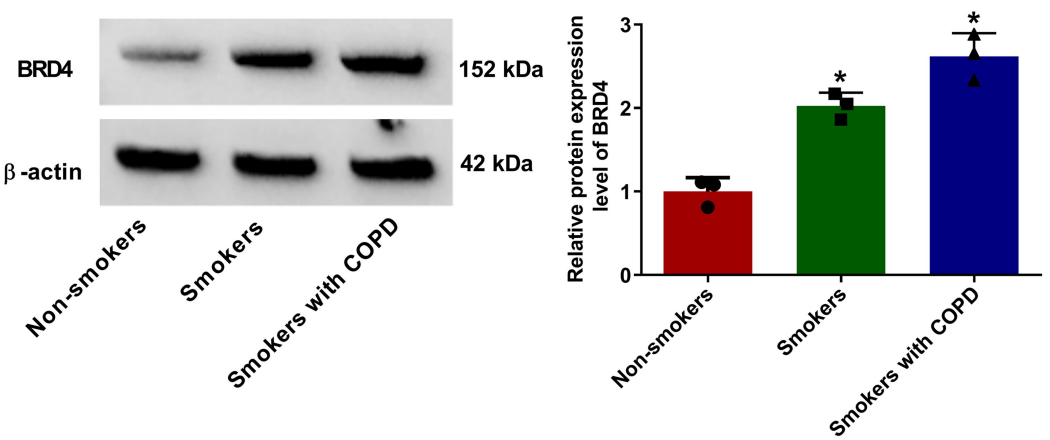

$\mathbf{F}$

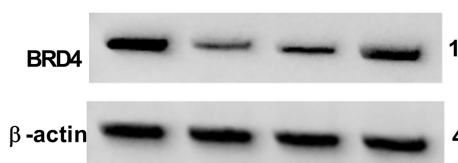

G

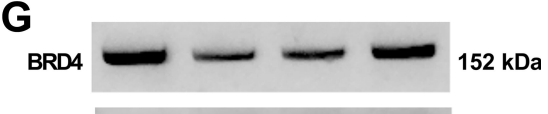

$42 \mathrm{kDa} \quad \beta$-actin $42 \mathrm{kDa}$
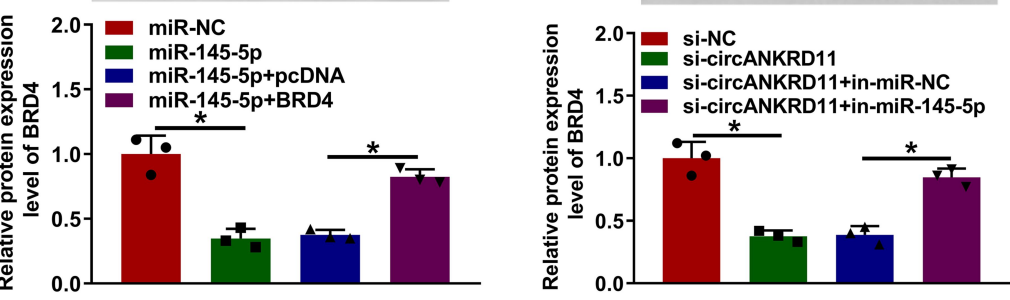

Figure 5 CircANKRDII modulated BRD4 via binding to miR-I45-5p. (A) The binding sites between miR-I45-5p and BRD4 were predicted by microT_CDS online database. (B) Luciferase activities were detected by dual-luciferase reporter assay in HPMECs. (C and D) The mRNA and protein expression of BRD4 were determined by qRT-PCR and Western blot, respectively, in the lung tissues of non-smokers ( $N=10)$, smokers without COPD (N=I7) and smokers with COPD (N=2I). (E) BRD4 protein expression was detected by Western blot analysis in HPMECs treated with $0 \%$ CSE or $2.5 \%$ CSE. (F) The effects between miR-145-5P mimics and BRD4 overexpression on the protein expression of BRD4 were determined by Western blot analysis in HPMECs. (G) The impacts between circANKRDII silencing and miR-I45-5P inhibitors on BRD4 protein expression were revealed by Western blot analysis in HPMECs. $* P<0.05$.

smokers with COPD as compared to smokers without COPD (Figure 5C and D). Additionally, BRD4 protein expression was also increased in $2.5 \%$ CSE-induced HPMECs when compared with untreated HPMECs (Figure 5E). The repressive impact of miR-145-5p mimics on BRD4 protein expression was also restored by BRD4 overexpression (Figure 5F). These results demonstrated BRD4 was a target gene of miR-145-5p. Furthermore, Western blot analysis presented that circANKRD11 knockdown decreased BRD4 protein expression, whereas miR-145-5p inhibitors reversed this impact (Figure $5 \mathrm{G}$ ), suggesting that circANKRD11 regulated BRD4 expression by associating with miR-145-5p.
MiR-I45-5p Mimics Suppressed Cell Apoptosis, Inflammation, and Oxidative Stress by Targeting BRD4 in CSE-Induced HPMECs

MiR-145-5p had been proved to bind to BRD4, whether it regulated CSE-aroused HPMEC injury by binding to BRD4 was further disclosed. To answer question, the effects between miR-145-5p mimics and BRD4 overexpression on cell apoptosis, inflammation, and oxidative stress were displayed. Results showed miR-145-5p mimics repressed cell apoptosis, but BRD4 overexpression attenuated this effect (Figure 6A). MiR-145-5p mimics also upregulated Bcl-2 

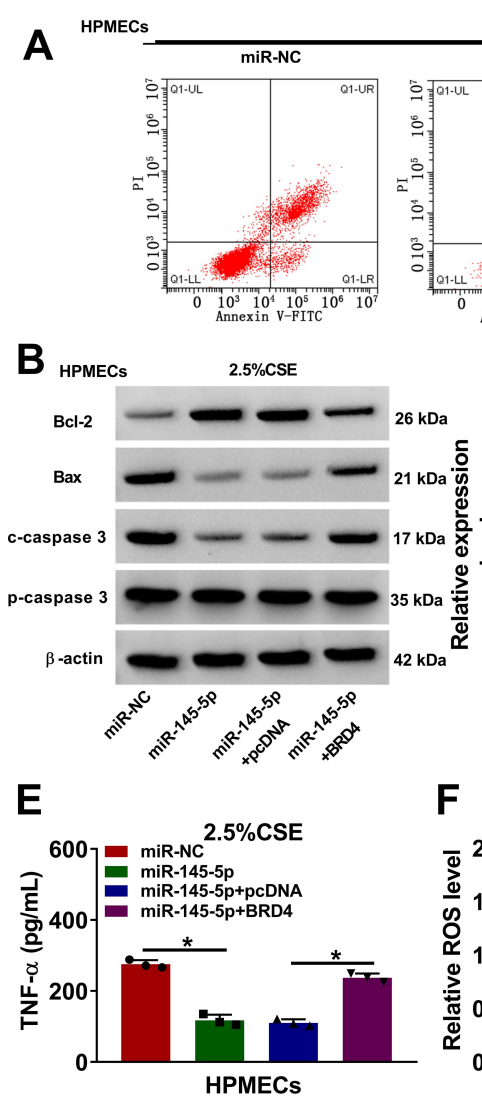

$\mathbf{F}$

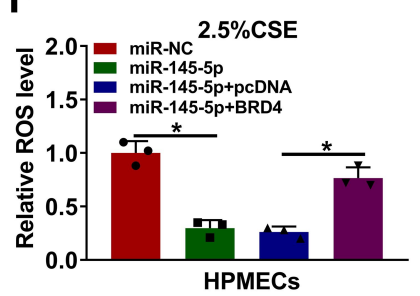

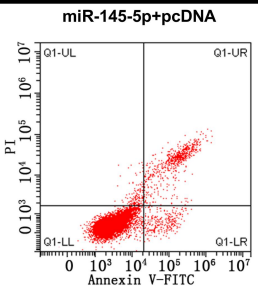

C

(1)

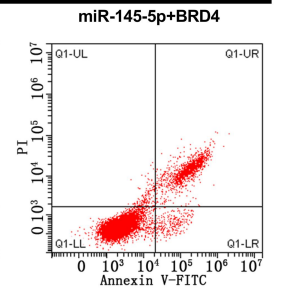

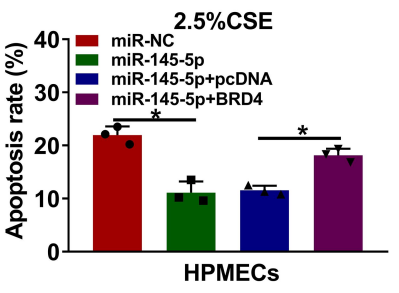

D

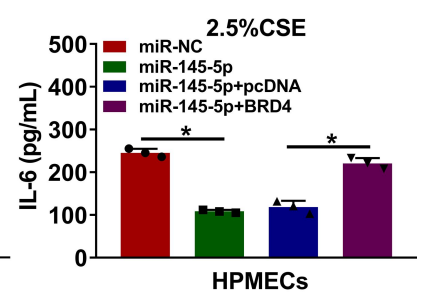

Figure 6 MiR-145-5p mimics protected against CSE-induced HPMEC injury through interacting with BRD4. (A-H) HPMECs were transfected with miR-NC, miR-I45-5p, miR-145-5p+pcDNA and miR-145-5p+BRD4, respectively, under 2.5\% CSE treatment in HPMECs. (A) Cell apoptosis was detected by Annexin V-FITC and PI double staining assay. (B) The protein expression of Bcl-2, Bax, c-caspase 3 and p-caspase 3 was determined by Western blot analysis. (C-E) The levels of IL-I $\beta$, IL-6 and TNF- $\alpha$ were detected by ELISA. (F) The level of ROS was detected by ROS detection assay. (G) MDA level was detected by MDA determination assay. (H) SOD activity was detected by SOD activity detection assay. $* P<0.05$.

protein expression, and downregulated the protein level of Bax and the value of c-caspase 3/p-caspase 3; however, these effects were reversed after transfection of BRD4 (Figure 6B). Additionally, the production of IL-1 $\beta$, IL-6 and TNF- $\alpha$ was inhibited by miR-145-5p mimics, which was impaired by ectopic BRD4 expression (Figure 6C-E). Furthermore, the levels of ROS and MDA were downregulated, and SOD activity was promoted after transfection of miR-145-5p, whereas these effects were reversed after BRD4 overexpression (Figure 6F-H). These results demonstrated miR-145-5p could modulate CSE-induced HPMEC injury via targeting BRD4.

\section{Discussion}

The features of circRNAs in structure and expression make them serve as diagnostic biomarkers or therapeutic targets for diseases. $^{22}$ In COPD, circRNAs have been seldom investigated previously. Current reports demonstrated that circ_0001859 could be employed as a diagnostic and prognostic biomarker based on its dysregulation in CS-treated lung tissues of mice and peripheral blood of patients with COPD as well as the analysis of receiver operating characteristics (ROC) curve. ${ }^{23}$ Duan et al analyzed the expression profiles of circRNAs and mRNAs, and demonstrated that circRNAs played vital roles in COPD process via regulating the immune balance and inflammatory responses. ${ }^{24}$ In mechanism, Ma and his colleagues explained that circ_0061052 implicated in epithelial-mesenchymal transition of CSE-induced airway remolding by controlling forkhead box $\mathrm{C} 1 /$ snail pathway through binding to miR-515-5p. ${ }^{25}$ Circ_0006872 was shown to facilitate CSE-triggered cell injury by mediating cell apoptosis, inflammatory response and oxidative stress via regulating miR-145-5p/nuclear factor kappaB pathway. ${ }^{26}$ In this paper, a novel circRNA, circANKRD11, was revealed that its silencing protected HPMECs from CSE-induced injury by miR-145-5p/BRD4 axis.

CS can induce cell apoptosis in various types of cells, such as fibroblasts and vascular endothelial cells. ${ }^{27,28} \mathrm{CS}$ is the bestdefined stimulant that causes COPD and leads to nonreversible pulmonary injury. $^{29}$ In this research, circANKRD11 was 
augmented in the lung tissue samples of smokers without COPD, and was greatly augmented in smokers with COPD. Another result presented that circANKRD11 expression was dose- and time-dependently upregulated in CSE-treated HPMECs. These evidences suggested that circANKRD11 might be enrolled in the regulation of smoke-related COPD process. Subsequently, loss-of function experiments displayed that CSE treatment induced the upregulation of apoptotic rate and inflammatory factors and promoted oxidative stress, but these effects were attenuated by reduced circANKRD11, implying circANKRD11 knockdown could restrain CSEinduced cell injury.

CircRNAs commonly served as competing endogenous RNAs to sponge miRNAs. ${ }^{30}$ So, the miRNA interacted with circANKRD11 was predicted. Our data presented miR-145$5 \mathrm{p}$ bound to circANKRD11, which was proved by qRT-PCR, dual-luciferase reporter, RIP and RNA pull-down assays. Current data presented that miR-145-5p had various roles in the process of diseases. For example, miR-145-5p could repress cell growth of glioma $^{31}$ and hepatocellular carcinoma, ${ }^{32}$ attenuated hypertrophic scar, ${ }^{33}$ regulated sensitivity of prolactinoma to bromocriptine ${ }^{34}$ and suppressed airway remodeling in CSE-triggered COPD. ${ }^{35}$ From our results, miR-145-5p was lowly expressed in smokers with or without COPD and CSE-induced HPMECs compared with non-smokers and untreated HPMECs, respectively, and miR-145-5p inhibitors restored circANKRD11 silencing-mediated effects on CSE-induced cell injury, meaning miR-145-5p could inhibit cell apoptosis, inflammation and oxidative stress, which was further proved by the previous data. ${ }^{19,26}$ Besides, these results explained that circANKRD11 regulated CSE-induced cell injury by sponging miR-145-5p.

Next, the target gene of miR-145-5p was continued to be sought. BRD4 was identified as an interest gene of miR-145$5 p$ through dual-luciferase reporter assay. BRD4 has been revealed to repress the production of IL- $1 \beta$-caused inflammatory factors in human airway epithelial cells. ${ }^{36}$ Liu et al showed BRD4 downregulation could protect mice from LPS-induced COPD by blocking NF- $\mathrm{B}$ pathway. ${ }^{37} \mathrm{Of}$ note, Castellucci et al indicated that IL-10 could repress LPStriggered expression of TNF- $\alpha$ and $\mathrm{C}-\mathrm{X}-\mathrm{C}$ motif chemokine ligand 8 (CXCL8) by damaging BRD4-dokjing sites. ${ }^{38}$ Additionally, BRD4 was also found to regulate CSEtriggered cell proliferation, apoptosis and inflammatory response in BEAS-2B cells. ${ }^{39}$ In this paper, data also presented that BRD4 was augmented in the lung tissues of smokers with or without COPD as well as CSE-treated HPMECs, and that BRD4 induced cell apoptosis as well as promoted inflammation and oxidative stress, which were in line with the study of Song et al. ${ }^{20}$ In the meantime, the repressive impacts of BRD4 overexpression on miR-145-5pmediated action implied that miR-145-5p regulated CSEinduced cell injury by targeting BRD4. Further, rescue experiments displayed that circANKRD11 regulated BRD4 expression by binding to miR-145-5p.

All in all, circANKRD11 knockdown mitigated CSEinduced cell apoptosis, inflammatory response and oxidative stress in HPMECs. CircANKRD11 was proved to bind to miR-145-5p, which was further found to target BRD4. Additionally, circANKRD11 knockdown attenuated CSE-induced cell injury by binding to miR-145-5p. And miR-145-5p mimics ameliorated CSE-caused cell damage via interacting with BRD4. Furthermore, circANKRD11 was presented to regulate BRD4 through associating with miR-145-5p. Collectively, circANKRD11 regulated CSE-induced HPMEC injury by controlling BRD4 through sponging miR-145-5p (as shown in Figure 7). This study not only demonstrates that circANKRD11 has the potential to serve as a diagnostic biomarker for smoke-related COPD, but also provides a theoretical basis for studying circRNA-directed therapy for smoke-caused COPD.

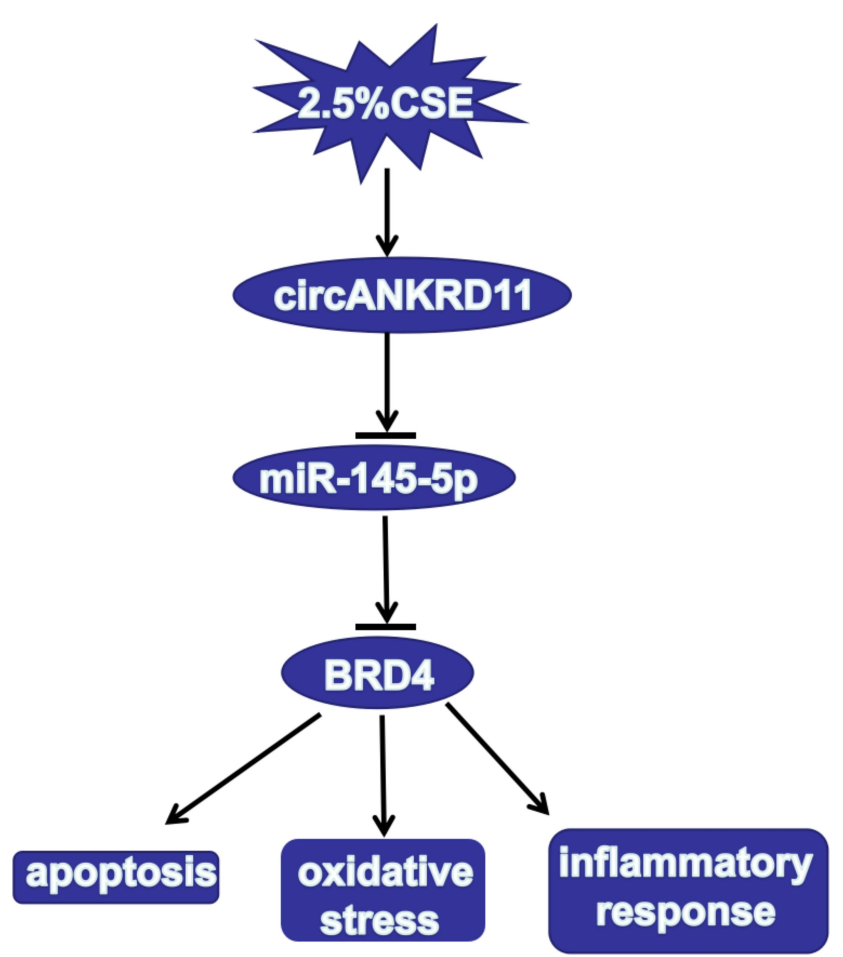

Figure 7 The illustration of circANKRDII in regulating CSE-induced cell injury of HPMECs. CircANKRDII regulated CSE-induced cell apoptosis, oxidative stress and inflammatory reaction by controlling BRD4 expression by sponging miR-145-5p. 


\section{Funding}

This work was approved by People's livelihood science and technology project, Key Research and Development Project in Hebei province in 2019 (19277760D); Medical science research project of hebei province in 2019 (20190524); General Program of Natural Science Foundation of Hebei(H2019206263).

\section{Disclosure}

The authors declare that they have no financial conflicts of interest.

\section{References}

1. Vogelmeier CF, Criner GJ, Martinez FJ, et al. Global strategy for the diagnosis, management, and prevention of chronic obstructive lung disease 2017 report: GOLD executive summary. Eur Respir J. 2017;49(3):1700214. doi:10.1183/13993003.00214-2017

2. Salimian J, Mirzaei H, Moridikia A, et al. Chronic obstructive pulmonary disease: microRNAs and exosomes as new diagnostic and therapeutic biomarkers. J Res Med Sci. 2018;23:27. doi:10.4103/jrms. JRMS_1054_17

3. Tuder RM, Petrache I. Pathogenesis of chronic obstructive pulmonary disease. J Clin Invest. 2012;122(8):2749-2755. doi:10.1172/ JCI60324

4. Chen Y, Luo H, Kang N, et al. Beraprost sodium attenuates cigarette smoke extract-induced apoptosis in vascular endothelial cells. Mol Biol Rep. 2012;39(12):10447-10457. doi:10.1007/s11033-012-1924-1

5. Arunachalam G, Yao H, Sundar IK, et al. SIRT1 regulates oxidantand cigarette smoke-induced eNOS acetylation in endothelial cells: role of resveratrol. Biochem Biophys Res Commun. 2010;393 (1):66-72. doi:10.1016/j.bbrc.2010.01.080

6. Ji W, Qiu C, Wang M, et al. Hsa_circ_0001649: a circular RNA and potential novel biomarker for colorectal cancer. Biochem Biophys Res Commun. 2018;497(1):122-126. doi:10.1016/j.bbrc.2018.02.036

7. Rong D, Sun H, Li Z, et al. An emerging function of circRNA-miRNAs-mRNA axis in human diseases. Oncotarget. 2017;8(42):73271-73281. doi:10.18632/oncotarget.19154

8. Li R, Jiang J, Shi H, et al. CircRNA: a rising star in gastric cancer. Cell Mol Life Sci. 2020;77(9):1661-1680. doi:10.1007/s00018-01903345-5

9. Guo R, Zhang L, Meng J. Circular RNA ANKRD36 attends to lipopolysaccharide-aroused MRC-5 cell injury via regulating microRNA-31-3p. Biofactors. 2020;46(3):391-401. doi:10.1002/ biof. 1592

10. Huang Z, Su R, Qing C, et al. Plasma circular RNAs hsa_circ_0001953 and hsa_circ_0009024 as diagnostic biomarkers for active tuberculosis. Front Microbiol. 2018;9:2010. doi:10.3389/ fmicb.2018.02010

11. Zhou S, Jiang H, Li M, et al. Circular RNA hsa_circ_0016070 is associated with pulmonary arterial hypertension by promoting PASMC proliferation. Mol Ther Nucleic Acids. 2019;18:275-284. doi:10.1016/j.omtn.2019.08.026

12. Chen D, Ma W, Ke Z, et al. CircRNA hsa_circ_100395 regulates miR-1228/TCF21 pathway to inhibit lung cancer progression. Cell Cycle. 2018;17(16):2080-2090. doi:10.1080/ 15384101.2018.1515553

13. Chen T, Yang Z, Liu C, et al. Circ_0078767 suppresses non-smallcell lung cancer by protecting RASSF1A expression via sponging miR-330-3p. Cell Prolif. 2019;52(2):e12548. doi:10.1111/cpr.12548
14. Molina-Pinelo S, Pastor MD, Suarez R, et al. MicroRNA clusters: dysregulation in lung adenocarcinoma and COPD. Eur Respir J. 2014;43(6):1740-1749. doi:10.1183/09031936.00091513

15. Stolzenburg LR, Harris A. The role of microRNAs in chronic respiratory disease: recent insights. Biol Chem. 2018;399(3):219-234. doi:10.1515/hsz-2017-0249

16. Diao X, Zhou J, Wang S, et al. Upregulation of miR-132 contributes to the pathophysiology of COPD via targeting SOCS5. Exp Mol Pathol. 2018;105(3):285-292. doi:10.1016/j.yexmp.2018.10.002

17. Shen W, Liu J, Fan M, et al. MiR-3202 protects smokers from chronic obstructive pulmonary disease through inhibiting FAIM2: an in vivo and in vitro study. Exp Cell Res. 2018;362(2):370-377. doi:10.1016/j.yexcr.2017.11.038

18. Belkina AC, Denis GV. BET domain co-regulators in obesity, inflammation and cancer. Nat Rev Cancer. 2012;12(7):465-477. doi:10.1038/nrc3256

19. Dang X, Yang L, Guo J, et al. miR-145-5p is associated with smoke-related chronic obstructive pulmonary disease via targeting KLF5. Chem Biol Interact. 2019;300:82-90. doi:10.1016/j.cbi.2019. 01.011

20. Song J, Wang Q, Zong L. LncRNA MIR155HG contributes to smoke-related chronic obstructive pulmonary disease by targeting miR-128-5p/BRD4 axis. Biosci Rep. 2020;40(3):BSR20192567. doi:10.1042/BSR20192567

21. Richter A, O'donnell RA, Powell RM, et al. Autocrine ligands for the epidermal growth factor receptor mediate interleukin-8 release from bronchial epithelial cells in response to cigarette smoke. Am J Respir Cell Mol Biol. 2002;27(1):85-90. doi:10.1165/ajrcmb.27.1.4789

22. Świtlik WZ, Karbownik MS, Suwalski M, et al. Serum miR-210-3p as a potential noninvasive biomarker of lung adenocarcinoma: a preliminary study. Genet Test Mol Biomarkers. 2019;23 (5):353-358. doi:10.1089/gtmb.2018.0275

23. Chen S, Yao Y, Lu S, et al. CircRNA0001859, a new diagnostic and prognostic biomarkers for COPD and AECOPD. BMC Pulm Med. 2020;20(1):311. doi:10.1186/s12890-020-01333-1

24. Duan R, Niu H, Yu T, et al. Identification and bioinformatic analysis of circular RNA expression in peripheral blood mononuclear cells from patients with chronic obstructive pulmonary disease. Int J Chron Obstruct Pulmon Dis. 2020;15:1391-1401. doi:10.2147/COPD.S252896

25. Ma H, Lu L, Xia H, et al. Circ0061052 regulation of FoxC1/Snail pathway via miR-515-5p is involved in the epithelial-mesenchymal transition of epithelial cells during cigarette smoke-induced airway remodeling. Sci Total Environ. 2020;746:141181. doi:10.1016/j. scitotenv.2020.141181

26. Xue M, Peng N, Zhu X, et al. Hsa_circ_0006872 promotes cigarette smoke-induced apoptosis, inflammation and oxidative stress in HPMECs and BEAS-2B cells through the miR-145-5p/NF-kappaB axis. Biochem Biophys Res Commun. 2021;534:553-560.

27. Kim YJ, Kim JY, Yoon JY, et al. Protective effect of aminophylline against cigarette smoke extract-induced apoptosis in human lung fibroblasts (MRC-5 cells). Basic Clin Pharmacol Toxicol. 2011;109 (1):17-22. doi:10.1111/j.1742-7843.2011.00673.x

28. Long YJ, Liu XP, Chen SS, et al. miR-34a is involved in CSE-induced apoptosis of human pulmonary microvascular endothelial cells by targeting Notch-1 receptor protein. Respir Res. 2018;19 (1):21. doi:10.1186/s12931-018-0722-2

29. Thomsen M, Ingebrigtsen TS, Marott JL, et al. Inflammatory biomarkers and exacerbations in chronic obstructive pulmonary disease. JAMA. 2013;309(22):2353-2361. doi:10.1001/jama.2013.5732

30. Dong Y, He D, Peng Z, et al. Circular RNAs in cancer: an emerging key player. J Hematol Oncol. 2017;10(1):1-8. doi:10.1186/s13045016-0370-2

31. Chen J, Chen T, Zhu Y, et al. circPTN sponges miR-145-5p/miR-330$5 \mathrm{p}$ to promote proliferation and stemness in glioma. J Exp Clin Cancer Res. 2019;38(1):398. doi:10.1186/s13046-019-1376-8 
32. Liang H, Sun H, Yang J, et al. miR-145-5p reduces proliferation and migration of hepatocellular carcinoma by targeting KLF5. Mol Med Rep. 2018;17(6):8332-8338. doi:10.3892/mmr.2018.8880

33. Shen W, Wang Y, Wang D, et al. miR-145-5p attenuates hypertrophic scar via reducing Smad2/Smad3 expression. Biochem Biophys Res Commun. 2020;521(4):1042-1048. doi:10.1016/j.bbrc.2019.11.040

34. Jian M, Du Q, Zhu D, et al. Tumor suppressor miR-145-5p sensitizes prolactinoma to bromocriptine by downregulating TPT1. $J$ Endocrinol Invest. 2019;42(6):639-652. doi:10.1007/s40618-0180963-4

35. Gu W, Yuan Y, Wang L, et al. Long non-coding RNA TUG1 promotes airway remodelling by suppressing the miR-145-5p/DUSP6 axis in cigarette smoke-induced COPD. J Cell Mol Med. 2019;23 (11):7200-7209. doi:10.1111/jcmm.14389

36. Khan YM, Kirkham P, Barnes PJ, et al. Brd4 is essential for IL-1 $\beta$ induced inflammation in human airway epithelial cells. PLoS One. 2014;9(4):e95051. doi:10.1371/journal.pone.0095051
37. Liu Y, Huang ZZ, Min L, et al. The BRD4 inhibitor JQ1 protects against chronic obstructive pulmonary disease in mice by suppressing NF- $\kappa$ B activation. Histol Histopathol. 2020;18283.

38. Castellucci M, Rossato M, Calzetti F, et al. IL-10 disrupts the Brd4docking sites to inhibit LPS-induced CXCL8 and TNF- $\alpha$ expression in monocytes: implications for chronic obstructive pulmonary disease. J Allergy Clin Immunol. 2015;136(3):781-791.e789. doi:10.1016/j.jaci.2015.04.023

39. Song B, Wu S, Ye L, et al. LncRNA TUG1 knockdown mitigates inflammatory injury induced by cigarette smoke extract in chronic obstructive pulmonary disease via miR-34c/BRD4 axis. Biosci Rep. 2020. doi:10.1042/BSR20193896

\section{Publish your work in this journal}

The International Journal of COPD is an international, peer-reviewed journal of therapeutics and pharmacology focusing on concise rapid reporting of clinical studies and reviews in COPD. Special focus is given to the pathophysiological processes underlying the disease, intervention programs, patient focused education, and self management protocols. This journal is indexed on PubMed Central, MedLine and CAS. The manuscript management system is completely online and includes a very quick and fair peer-review system, which is all easy to use. Visit http://www.dovepress.com/testimonials.php to read real quotes from published authors. 